\title{
Propagation in the atmosphere of ultrahigh-energy charmed hadrons
}

\author{
R. Barceló, J.I. Illana, M.D. Jenkins, M. Masip \\ CAFPE and Departamento de Física Teórica y del Cosmos \\ Universidad de Granada, E-18071, Granada, Spain \\ rbarcelo@ugr.es, jillana@ugr.es, mjenk@ugr.es, masip@ugr.es
}

\begin{abstract}
Charmed mesons may be produced when a primary cosmic ray or the leading hadron in an air shower collide with an atmospheric nucleon. At energies $\geq 10^{8} \mathrm{GeV}$ their decay length becomes larger than $10 \mathrm{~km}$, which implies that they tend to interact in the air instead of decaying. We study the collisions of long-lived charmed hadrons in the atmosphere. We show that $\left(\Lambda_{c}, D\right)$-proton diffractive processes and partonic collisions of any $q^{2}$ where the charm quark is an spectator have lower inelasticity than $(p, \pi)$-proton collisions. In particular, we find that a $D$ meson deposits in each interaction just around 55\% of the energy deposited by a pion. On the other hand, collisions involving the valence $c$ quark (its annihilation with a sea $\bar{c}$ quark in the target or $c$-quark exchange in the $t$ channel) may deposit most of the $D$ meson energy, but their frequency is low (below $0.1 \%$ of inelastic interactions). As a consequence, very energetic charmed hadrons may keep a significant fraction of their initial energy after several hadronic interactions, reaching much deeper in the atmosphere than pions or protons of similar energy.
\end{abstract}




\section{Introduction}

Cosmic rays reach the Earth with energies of up to $10^{11} \mathrm{GeV}$. When they enter the atmosphere they experience ultrahigh energy collisions, probing a scale not accessible at colliders. These processes are interesting because they could produce exotic particles or involve interactions not seen at lower energies. There is, however, a second generic reason that make these cosmic-ray collisions interesting: they probe a regime where the properties of the standard particles may be substantially different.

In particular, here we will focus on the charm quark. Charm-quark pairs may be abundant inside extensive air showers [1, 2]. Once produced, each quark will result into a $D^{+, 0}$ meson or a $\Lambda_{c}^{+}$baryon, that will then decay weakly into light hadrons and (with a $\approx 0.1$ branching ratio) leptons. These processes have been extensively studied in the literature, as they may be the dominant source of atmospheric muons and neutrinos of energy $E \geq 10^{6} \mathrm{GeV}$ [3, 4, [5, 6]. A new regime, however, would be achieved if the charmed hadron has an energy $E>E_{c}$ such that its decay length $\left(\lambda_{d}=c \tau E / m\right)$ becomes larger than its interaction length $\left(\lambda_{i}\right)$ in the atmosphere. For example, a $10^{8} \mathrm{GeV} D^{+}$meson is effectively a long-lived $\left(\lambda_{d}^{D^{+}} \approx 18\right.$ $\mathrm{km}$ ) heavy hadron that interacts and propagates in the air instead of decaying. Such a behaviour, unknown of in colliders (where a $D$ meson decays before it has experienced any type of interaction) could look somewhat similar to the one of the $R$-hadrons predicted in some supersymmetric models [7, 8]. The propagation in matter of a charmed hadron is of no interest at the Tevatron or the LHC, but it is most likely important in extensive air-shower experiments.

In this article we analyze the collisions of very energetic $D$ mesons and $\Lambda_{c}^{+}$baryons with protons at rest. Our objective is to estimate the amount of energy that they deposit in each hadronic interaction in the atmosphere. First we study diffractive processes, using the Montecarlo code PYTHIA [9] to simulate the collisions. Then we consider partonic collisions of any $q^{2}$, with our analysis also based on PYTHIA. Finally we estimate the effects of going from a proton to a nucleus target.

\section{Diffractive processes}

A charmed hadron $H$ is basically different from a pion or a proton in the fact that it contains a heavy core of mass $m_{c} \approx 1.27 \mathrm{GeV}$. If one thinks of a proton as three clouds of mass $m=0.3 \mathrm{GeV}$ associated to the three constituent quarks, then the $\Lambda_{c}$ baryon consists also of three similar clouds, but one of them has an additional electroweak core and a total 
mass $m_{c}+m=1.6 \mathrm{GeV}$. In a long-distance (low- $\left.q^{2}\right)$ collision with an atmospheric nucleon, however, this heavy core will be invisible (the proton and the $\Lambda_{c}$ clouds will look identical), as only interactions of $q^{2} \geq m_{c}^{2}$ can resolve it. Therefore, we will assume that the momentum exchanged through pomerons or other non-perturbative dynamics with the target does not depend on the electroweak core in $H$.

Let us be more specific. We separate the mass $m_{H}$ of the heavy hadron into

$$
m_{H}=m_{c}+\Lambda
$$

The light degrees of freedom in $H$ carry just a fraction $w \equiv\left(m_{H}-m_{c}\right) / m_{H}$ of the hadron energy $E$, so in a diffractive process $H$ will be seen by the target nucleon like a light hadron of energy $w E$. Therefore, to estimate the momentum $q^{\mu}$ absorbed by $H$ in the process, we will just simulate with PYTHIA the collision of a proton (for $H=\Lambda_{c}$ ) or a pion (for $H=D$ ) of energy $w E$ with the nucleon and will assume that $q^{\mu}$ is the same when the incident particle is the charmed hadron.

Once $H$ absorbs the momentum $q^{\mu}$ it becomes a diffractive system $H_{\text {dif }}$ of mass $M$, the critical parameter in the collision. If $M<m_{H}+1 \mathrm{GeV}$ the collision is quasielastic and $H_{\text {dif }}$ will just decay into two bodies $(e . g ., H+\eta)$. For larger values of $M$ the system is treated by PYTHIA [10] like a string with the quantum numbers of $H$. When $H$ is a baryon the string may be stretched between a quark and a diquark or between a quark, a gluon and a diquark, whereas for a diffractive meson the string connects a quark and an antiquark or a quark, a gluon and an antiquark.

To illustrate our procedure, let us consider the diffractive collision of a $10^{9} \mathrm{GeV} \Lambda_{c}^{+}$ baryon $\left(m_{\Lambda_{c}}=2.28 \mathrm{GeV}\right)$ with a proton $\left(m_{p}=0.94 \mathrm{GeV}\right)$ at rest. We need to simulate the collision of a $4.43 \times 10^{8} \mathrm{GeV}$ proton, and we will do it in the c.o.m. frame, where each proton carries $14.4 \mathrm{TeV}$.

(i) In a first PYTHIA example the diffracted proton absorbs $q^{\mu}=(-1.3 ;-0.13,0.10,-1.3)$ $\mathrm{GeV}$, getting a mass of $M=1.58 \mathrm{GeV}$. The system then decays into a $\Delta^{+} \pi^{0}$, and the $\Delta^{+}$finally produces a proton and another $\pi^{0}$. Going back to the lab frame we find the final proton with $E_{p}^{\prime}=2.75 \times 10^{8} \mathrm{GeV}$, i.e., the leading baryon carries a fraction 0.62 of the initial energy. The elasticity $z$ in this collision is then $z=0.62$. If the incident particle is a $10^{9} \mathrm{GeV} \Lambda_{c}^{+}$, we first go to the same $p p$ c.o.m. frame (where the energies of the charmed baryon and the target proton are $32.5 \mathrm{TeV}$ and $14.4 \mathrm{TeV}$, respectively). The $\Lambda_{c}^{+}$absorbs there the same momentum $q^{\mu}$ and gets a diffractive mass of $M=3.2$ $\mathrm{GeV}$, it then goes into $\Sigma_{c}^{+} \pi^{0}$, and the $\Sigma_{c}$ decays into a $\Lambda_{c}^{+}$and another $\pi^{0}$. Going back to the lab frame we obtain that the final $\Lambda_{c}^{+}$baryon carries $E^{\prime}=9.3 \times 10^{8} \mathrm{GeV}$, 
implying an elasticity $z=0.93$. This type of quasielastic processes accounts for $8 \%$ of all diffractive collisions.

(ii) In a second PYTHIA simulation the momentum absorbed by the incident proton in the c.o.m. frame is $q^{\mu}=(0.12 ; 0.14,0.14,-0.12) \mathrm{GeV}$, which produces a diffractive mass $M=83 \mathrm{GeV}$. The system evolves into a string stretching between a $u$-quark and a $(u d)$-diquark. Quark fragmentation and the (strong or electromagnetic) decay of baryonic resonances results then into a leading baryon plus 18 other hadrons of lower energies. In particular, in the lab frame there is a $p$ of $1.8 \times 10^{8} \mathrm{GeV}$ (i.e., $z=0.41$ ) plus 16 mesons (pions and kaons) and a $p \bar{n}$ pair sharing the rest of the energy. Changing the proton for a $10^{9} \mathrm{GeV} \Lambda_{c}^{+}$we obtain a diffractive mass of $M=124 \mathrm{GeV}$. Now the system may define 3 different (equally probable) diquark-quark strings: $(c d)-u$; $(c u)-d$ or $(u d)-c$. The first two cases tend to result into a leading charmed baryon after the collision. For example, in the first case we obtain a final $\Lambda_{c}^{+}$of $6.0 \times 10^{8} \mathrm{GeV}(z=0.60)$. The third case, however, is basically different, as the struck quark is the charm and it will most likely fragment into a $D$ meson. With the PYTHIA simulation we obtain a final $D^{+}$of $3.8 \times 10^{8} \mathrm{GeV}(z=0.38)$. In around $30 \%$ of diffractive collisions the $\Lambda_{c}$ changes into a $D$ meson. In contrast, in a diffractive proton-proton collision the leading hadron becomes a meson in just $15 \%$ of the cases.

(iii) In the final example PYTHIA provides an event with large diffractive mass $(M=6087$ $\left.\mathrm{GeV} ; q^{\mu}=(641 ; 0.36,0.43,-641) \mathrm{GeV}\right)$ and a diquark-gluon-quark string. The final spectrum is similar to the one in case (ii), although with a larger multiplicity of final states and a lower energy in the leading hadron. Whereas in the $p p$ collision we find a neutron carrying a $38 \%$ of the initial energy, in the $\Lambda_{c} p$ process we obtain a $4.1 \times 10^{8}$ $\mathrm{GeV}(z=0.41) \Lambda_{c}^{+}$or a $7.3 \times 10^{8} \mathrm{GeV}(z=0.73) D^{0}$ depending on the flavor of the struck quark.

In Fig. 1 we plot the distribution of the fraction $z$ of energy taken by the leading baryon after a $p p$ diffractive collision or by the charmed hadron after the analogous $\Lambda_{c} p$ process. The average values of the distributions are $\langle z\rangle=0.60$ and $\langle z\rangle=0.77$, respectively, which imply an inelasticity $K=1-\langle z\rangle$ :

$$
K_{p p}^{\text {dif }}=0.40 ; \quad K_{\Lambda_{c} p}^{\text {dif }}=0.23 .
$$

The regions of highest $z$ (with $K_{\Lambda_{c} p}^{(1)}=0.01$ ) correspond to $28 \%$ of diffractive processes where the incident hadron does not break (but the target proton does), whereas $8 \%$ of processes correspond to quasielastic events with $M<m_{\Lambda_{c}}+1 \mathrm{GeV}$ and have a slightly higher inelasticity, $K_{\Lambda_{c} p}^{(2)}=0.09 \pm 0.04$, where $\Delta K=\sqrt{\left\langle z^{2}\right\rangle-\langle z\rangle^{2}}$. In the remaining collisions the 


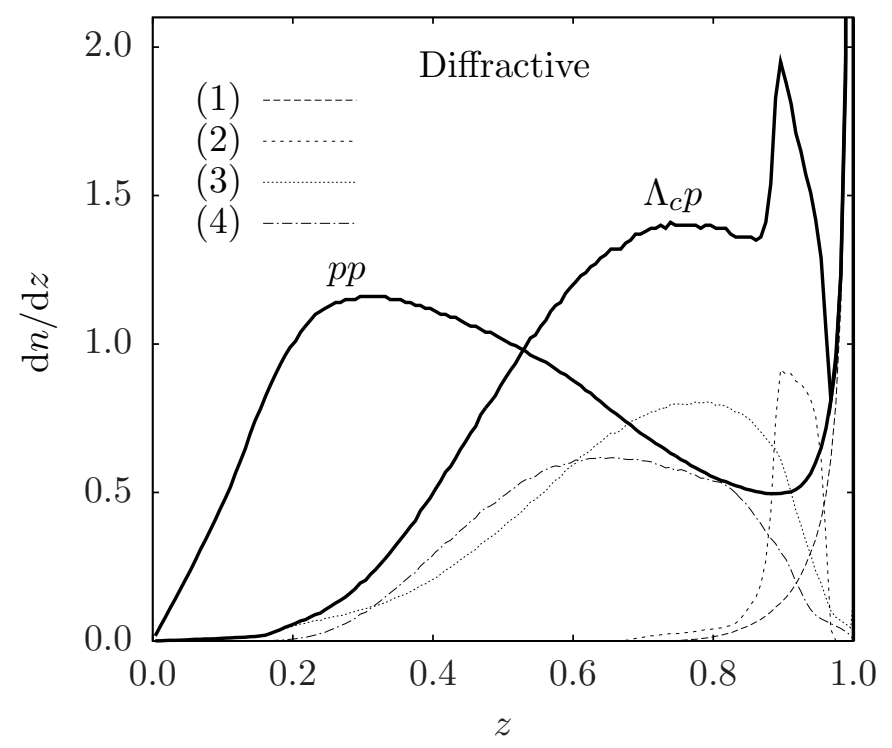

Figure 1: Fraction $z$ of energy taken by the leading baryon or the charmed hadron in $p p$ and $\Lambda_{c} p$ diffractive collisions, respectively. We have separated the distributions in the 4 types of $\Lambda_{c} p$ processes described in the text.

$\Lambda_{c}$ may become a $D$ meson or a charmed baryon (29\% and $35 \%$ of all diffractive processes, respectively). The $D$ mesons carry a fraction $\langle z\rangle=0.64$ of the initial energy $\left(i . e ., K_{\Lambda_{c} p}^{(3)}=\right.$ $0.36 \pm 0.16$ ), whereas charmed baryons imply a slightly lower inelasticity, $K_{\Lambda_{c} p}^{(4)}=0.32 \pm 0.17$. The leading $D$ meson may be a $D^{+}$, a $D^{0}$ or a $D_{s}$ in an approximate proportion of $1: 3: 0.5$ (the $D^{+}{ }^{0} D^{0}$ isospin symmetry is broken by the decay of charm resonances), whereas the baryons are mostly $\Lambda_{c}^{+}(94 \%)$ with some $\Xi_{c}^{0}(3 \%)$ and $\Xi_{c}^{+}(3 \%)$. In Fig. 1 we have separated these four types of diffractive $\Lambda_{c} p$ processes.

For an incident $10^{9} \mathrm{GeV} D^{+}$meson we proceed in an analogous way, studying the diffractive collision of a $3.2 \times 10^{8} \mathrm{GeV}$ pion with a proton at rest. We simulate with PYTHIA the collision in the c.o.m. frame, we read the $q^{\mu}$ absorbed by the pion and assume that the momentum absorbed by a $D^{+}$in that frame would be the same. We find that in $24 \%$ of the processes the incident meson remains unbroken (i.e., $M=m_{D}$ ), whereas quasielastic events $\left(M<m_{D}+1 \mathrm{GeV}\right)$ account for $15 \%$ of the total. The inelasticity of these two types of events is $K_{\pi p}^{(1)}=0.02$ and $K_{\pi p}^{(2)}=0.05 \pm 0.05$, respectively. The rest (61\%) of diffractive collisions deposit an average fraction $K_{\pi p}^{(3)}=0.33 \pm 0.17$ of the initial energy. In Fig. 2 we give the $z$ distribution for the three types of events. The peaks in $D$ collisions appear at $z \approx m_{D} /\left(m_{D}+m_{\eta, \omega}, \ldots\right)$, whereas the step in the pion distribution results from processes 


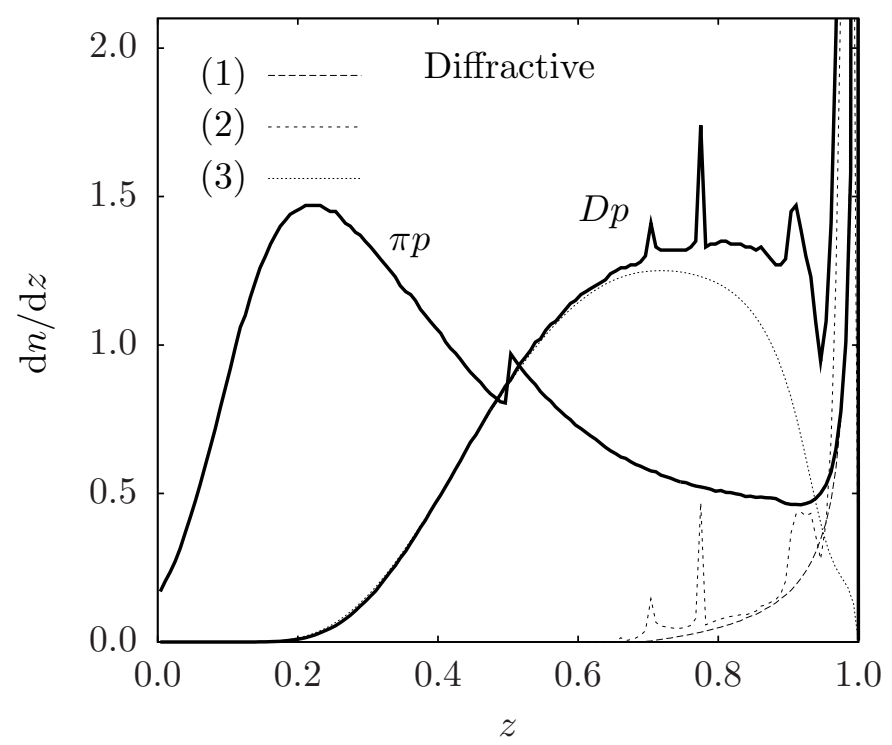

Figure 2: Fraction $z$ of energy taken by the leading baryon or the charmed hadron in $\pi p$ and $D p$ diffractive collisions, respectively. We have separated the distributions in the 3 types of $D p$ processes described in the text.

with two final pions of similar energy. The average inelasticity is

$$
K_{\pi p}^{d i f}=0.47 ; \quad K_{D p}^{d i f}=0.21
$$

Whereas a pion loses energy faster than a proton, the diffractive collisions of a $D$-meson are slightly less inelastic than the ones of a charmed baryon.

Finally, the diffractive cross section for a $10^{9} \mathrm{GeV}$ charmed hadron can be read from the PYTHIA estimate for a $4.4 \times 10^{8} \mathrm{GeV}$ proton or a $3.2 \times 10^{8} \mathrm{GeV}$ pion,

$$
\sigma_{\text {dif }}^{\Lambda_{c} p}=26.2 \mathrm{mb} ; \quad \sigma_{\text {dif }}^{D p}=15.6 \mathrm{mb}
$$

\section{Partonic collisions}

Non-diffractive processes dominate the inelastic cross section in PYTHIA simulations. In particular, at $4.43 \times 10^{8} \mathrm{GeV}$ the cross section for parton-parton interactions of any $q^{2}$ in $p p$ and $\pi p$ collisions is 1

$$
\sigma_{n-\text { dif }}^{p p}=62.2 \mathrm{mb} ; \quad \sigma_{n-\text { dif }}^{\pi p}=43.6 \mathrm{mb} .
$$

\footnotetext{
${ }^{1}$ PYTHIA extrapolates to $q^{2}<2 \mathrm{GeV}$ using a minimum-biased method.
} 
We will use these simulations to obtain an approximate description of partonic $H p$ collisions.

We model $H$ as a system with the same parton content as the corresponding proton or pion but substituting a valence up quark $u_{0}$ for the charm quark. Like in diffractive processes, we will associate a charmed hadron $H$ of energy $E$ to a light hadron of energy $E\left(m_{H}-m_{c}\right) / m_{H}$. If $u_{0}$ carries a fraction $x$ of the proton or pion momentum we will change it for a $c$ with

$$
x_{c}=\frac{m_{c}}{m_{H}}+\frac{m_{H}-m_{c}}{m_{H}} x .
$$

In this way the excess of energy in $H$ is carried entirely by the charm quark, whereas the light partons in both hadrons $(H$ and $p$ or $\pi$ ) carry exactly the same amount of energy.

We will then distinguish two types of $\mathrm{Hp}$ non-diffractive collisions: those where the charm is an spectator (i.e., it is a light parton in $H$ who hits a parton in the target proton), and processes where the charm itself interacts. For the first one we will just simulate with PYTHIA the parton process using a light hadron and then substitute the spectator $u_{0}$ for the charm quark. Charm interactions, on the other hand, have a much smaller cross section,

$$
\sigma_{\text {cint }}^{H p}=0.8 \mathrm{mb}
$$

than the processes with an spectator charm quark,

$$
\sigma_{c s p e c}^{\Lambda_{c} p}=61.4 \mathrm{mb} ; \quad \sigma_{c s p e c}^{D p}=42.8 \mathrm{mb}
$$

but they imply collisions of higher inelasticity. In particular, there is the possibility that the $c$ quark in the incident $H$ hadron annihilates with a sea $\bar{c}$ in the target proton. The charmed hadron after such process has lost basically all its energy. These events, however, occur in just a fraction of all partonic processes,

$$
\sigma_{c \bar{c}}^{H p}=0.02 \mathrm{mb}
$$

Let us first describe $\Lambda_{c} p$ non-diffractive collisions. In Fig. 3 we plot the fraction $z$ of energy carried by the charmed hadron after the collision. We obtain that the leading hadron may be a baryon $(55 \%)$ or a $D$ meson $(45 \%)$. The baryon may be a $\Lambda_{c}^{+}$or, with much smaller frequencies, $\Xi_{c}^{0}, \Xi_{c}^{+}$or $\Omega_{c}^{0}$. The three meson species $\left(D^{+}, D^{0}, D_{s}\right)$ appear with approximate frequencies of $(1: 3: 0.5)$. In Fig. 3 we have separated the spectra in the two cases. The average fraction of energy taken by the baryons is $\langle z\rangle=0.60$ (i.e., $K_{\Lambda_{c} p}^{(5)}=0.40 \pm 0.17$ ), whereas for the mesons it is just $\langle z\rangle=0.47\left(K_{\Lambda_{c} p}^{(6)}=0.53 \pm 0.15\right)$. The events where the charm interacts with a light parton in the target proton are mostly included among the ones with a leading $D$ meson, and have an average elasticity of $\langle z\rangle=0.42$. Finally, in $0.03 \%$ of the partonic collisions the incident charm anihilates (it is traded by a spectator sea charm 


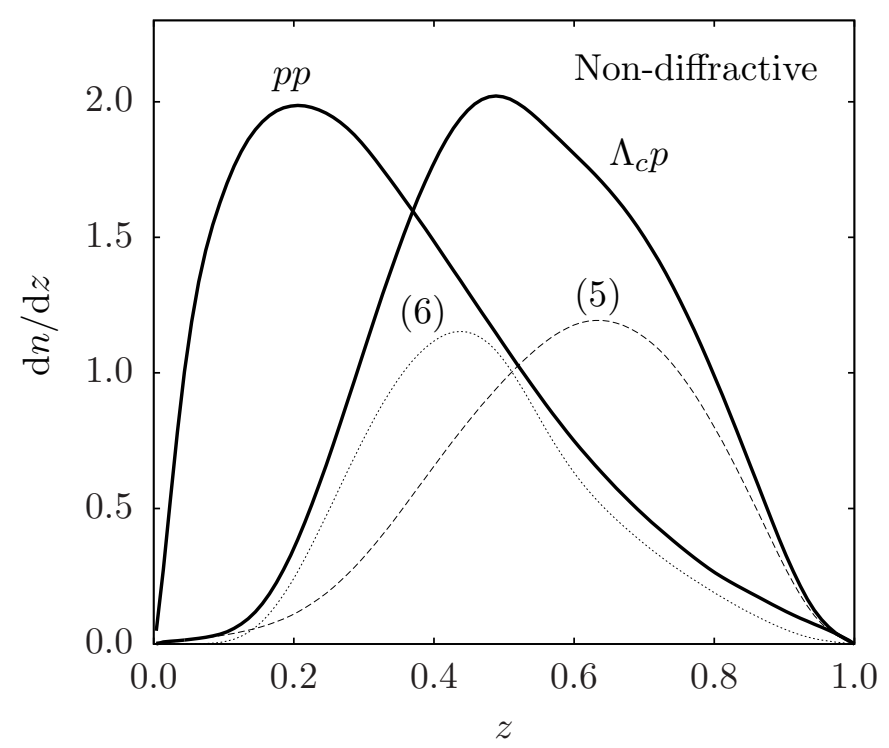

Figure 3: Fraction $z$ of energy taken by the leading baryon or the charmed hadron in $p p$ and $\Lambda_{c} p$ non-diffractive collisions, respectively. We have separated the distributions when the final state is a charmed baryon (5) or a charmed meson (6).

in the target proton) and $K_{\Lambda_{c} p}^{(7)} \approx 1$. The average inelasticity of non-diffractive collisions is (we include for comparison the inelasticity in $p p$ collisions)

$$
K_{p p}^{n-d i f}=0.66 ; \quad K_{\Lambda_{c} p}^{n-d i f}=0.46 .
$$

$D p$ non-diffractive collisions are summarized in Fig. 4. The final charm hadron is almost always (85\% of the events) a $D$ meson carrying a fraction $\langle z\rangle=0.55$ of the initial energy $\left(K_{D p}^{(4)}=0.45 \pm 0.16\right)$. The $c$ quark anihilates $\left(K_{D p}^{(5)} \approx 1\right)$ with a sea $\bar{c}$ in the target just in $0.04 \%$ of the $D p$ partonic collisions. The inelasticity in these non-diffractive processes is substantially lower than in pion collisions,

$$
K_{\pi p}^{n-d i f}=0.77 ; \quad K_{D p}^{n-d i f}=0.45 .
$$

\section{$4 \quad H$-nucleus collisions}

From the analysis in previous sections it results that the inelastic cross section for the collision of a $10^{9} \mathrm{GeV}$ charmed hadron with a proton at rest is

$$
\sigma^{\Lambda_{c} p}=88.4 \mathrm{mb} ; \quad \sigma^{D p}=59.2 \mathrm{mb},
$$




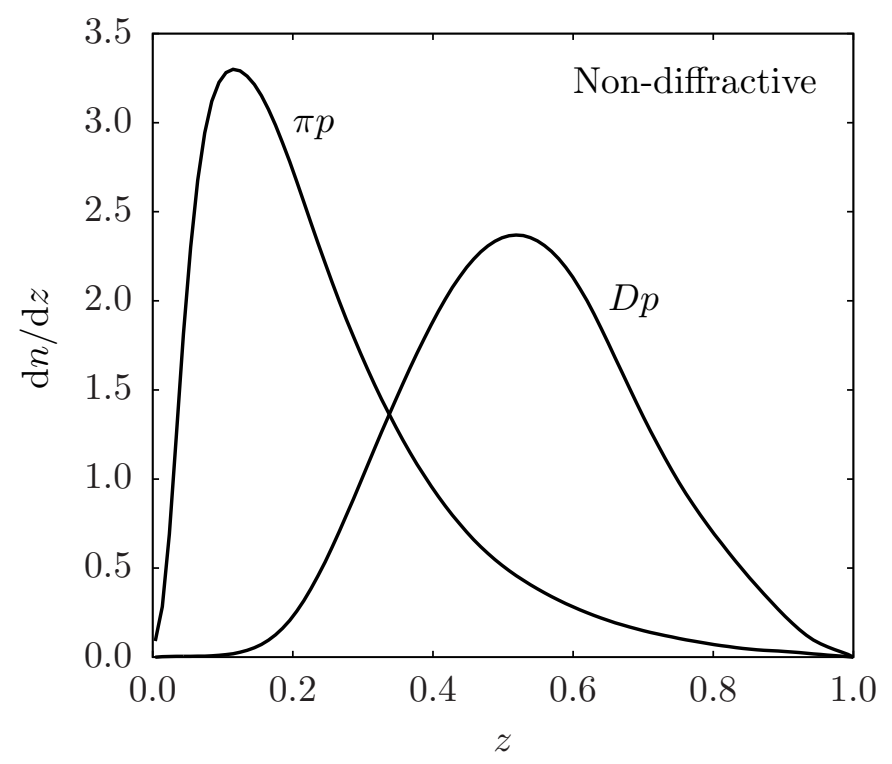

Figure 4: Fraction $z$ of energy taken by the leading baryon or the charmed hadron in $\pi p$ and $D p$ non-diffractive collisions, respectively.

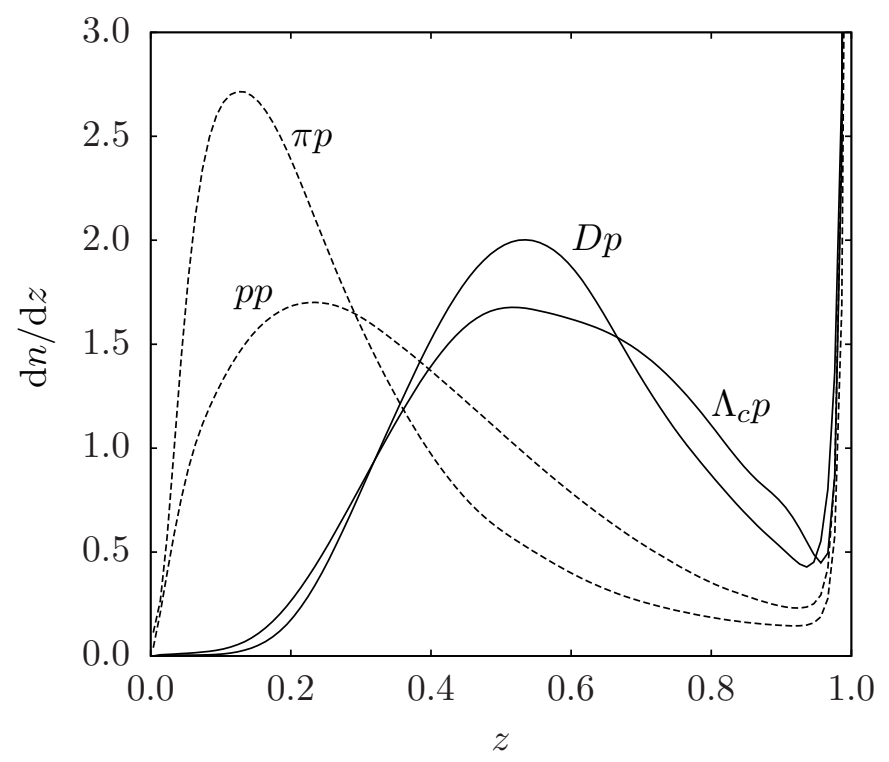

Figure 5: Fraction $z$ of energy taken by the leading baryon in $p p$ collisions, the leading pion in $\pi p$ collisions, and the charmed hadron in $\Lambda_{c} p$ and $D p$ collisions. The distributions include diffractive and partonic collisions. 
where diffractive processes contribute a $30 \%$ in $\Lambda_{c} p$ collisions and a $26 \%$ in $D p$ interactions. The cross section for proton and pion collisions of the same energy is around a $7 \%$ larger,

$$
\sigma^{p p}=94.1 \mathrm{mb} ; \quad \sigma^{\pi p}=64.8 \mathrm{mb} .
$$

The average inelasticity in these collisions is then

$$
K_{\Lambda_{c} p}=0.39 ; \quad K_{D p}=0.38,
$$

which is substantially lower than the one in proton and pion collisions,

$$
K_{p p}=0.59 ; \quad K_{\pi p}=0.70 .
$$

These results depend basically on the fragmentation model used by PYTHIA, so they should not change substantially if the analysis were based on the Montecarlo code SIBYLL [11] or on any other code using the Lund string scheme.

In this section we would like to comment on the approximate effects of going from a proton to a nucleus target. The total cross section for the collisions of $H$ with an atomic nucleus of mass number $A$ can be estimated as

$$
\sigma^{H A} \approx A^{2 / 3} \sigma^{H p},
$$

where the factor of $A^{2 / 3}$ takes into account the screening between the nucleons inside the nucleus. For an averaged atmospheric nucleus of $A=14.6$ this implies

$$
\sigma^{\Lambda_{c} a i r}=528 \mathrm{mb} ; \quad \sigma^{\text {Dair }}=354 \mathrm{mb} .
$$

The associated interaction length $\lambda_{\text {int }}^{H}=m_{\text {air }} / \sigma^{H \text { air }}$ in the atmosphere when $H$ is a charmed meson or a baryon is therefore

$$
\lambda_{\text {int }}^{\Lambda_{c}}=46 \mathrm{~g} / \mathrm{cm}^{2} ; \quad \lambda_{\text {int }}^{D}=69 \mathrm{~g} / \mathrm{cm}^{2},
$$

which is a $6 \%$ and a $9 \%$ longer than those of a pion and a proton of the same energy, respectively.

To deduce the spectrum of the leading hadron after the collision one must take into account that there may be more than one nucleon involved in each $H$-nucleus interaction. In general, it will be possible to distinguish between peripheral and central collisions. The first type is similar to the $H p$ processes discussed before, and we will then assume that the spectra coincide. Central collisions, on the other hand, imply a softer spectrum of secondaries and a smaller value of the fraction $z$ of energy taken by the leading charmed hadron. 
As a first order estimate, we will assume that peripheral and central collisions occur with equal frequency, and that the average inelasticity in central processes is the typical in a non-diffractive collision increased by $10 \%$ :

$$
K_{H a i r} \approx \frac{1}{2} K_{H \text { air }}^{\text {peri }}+\frac{1}{2} K_{H \text { air }}^{c e n t} \approx\left(\frac{1}{2} K_{H p}+\frac{1}{2} 1.1 K_{H p}^{n-d i f}\right) .
$$

At $E=10^{9} \mathrm{GeV}$ we obtain

$$
K_{\Lambda_{c} \text { air }} \approx 0.45 ; \quad K_{D \text { air }} \approx 0.44
$$

For proton and pion collisions the same prescription gives an inelasticity

$$
K_{\text {pair }} \approx 0.66 ; \quad K_{\text {aiir }} \approx 0.78 .
$$

This $12 \%$ increase in $K$ when going from a proton to a nucleus target compares well with the results obtained by other authors [12. The frequency of baryon to meson transitions in $\Lambda_{c}$-air central collisions would be close to the $45 \%$ obtained in partonic processes.

Finally, it is important to estimate the probability $p_{c \bar{c}}^{H}$ iir of a $c \bar{c}$ interaction that deposits all of the charmed hadron energy. Short-distance interactions in nucleus collisions scale proportional to the mass number, implying

$$
p_{c \bar{c}}^{H a i r} \approx \frac{A \sigma_{c \bar{c}}^{H p}}{A^{2 / 3} \sigma^{H p}} .
$$

We obtain

$$
p_{c \bar{c}}^{\Lambda_{c} a i r} \approx 0.0015 ; \quad p_{c \bar{c}}^{D a i r} \approx 0.0020
$$

\section{Summary and discussion}

Charmed hadrons that decay weakly become long lived in the atmosphere at energies $E>10^{8}$ $\mathrm{GeV}$, just like pions do at $E>10^{2} \mathrm{GeV}$. The behaviour of pions in a calorimeter is well understood and, as a consequence, their dynamics inside extensive air showers is also known. We know, for example, that ultrahigh energy pions are produced in these showers but never reach the ground, since the atmosphere is equivalent to 10 meters of water vertically and 30 times thicker from horizontal zenith angles. In contrast, $D$ mesons are not directly observable at calorimeters in colliders because they decay before they can reach them. Any estimate of their possible effects in air showers must then first model its hadronic interactions.

Here we have studied the collisions of charmed hadrons with protons. Our intention has not been to perform an analysis based on first principles, but to obtain an estimate of the 
qualitative features of such processes based on PYTHIA. The charm quark inside the hadron carries a large fraction $\left(m_{c} / m_{D} \approx 0.7\right)$ of energy, so one may expect that the spectrum of the final state will be substantially different from the one in pion-proton collisions. We have argued that in most of the processes either the charm can not be resolved $\left(q^{2} \ll m_{c}^{2}\right)$ or it is just an spectator. This allowed us to correlate them with pion or proton collisions, and use then PYTHIA to simulate quark fragmentation and the (strong or electromagnetic) decay of higher-mass resonances.

We obtain that the average inelasticity in $D p$ collisions is $K_{D p}=0.38$, a factor of 0.55 smaller than in $\pi p$ interactions. If the target is an air nucleus, we estimate values around $K_{\text {Dair }}=0.44$ and $K_{\pi \text { air }}=0.78$. These central values would imply that starting with an initial $10^{9} \mathrm{GeV}$ pion, after 10 hadronic interactions in the atmosphere one is left with leading meson of just $300 \mathrm{GeV}$. Instead, the energy of a $D$ meson would be reduced to $3 \times 10^{6} \mathrm{GeV}$.

In contrast to pions and protons, we find that the collisions of charmed mesons and baryons have a very similar inelasticity. However, $\Lambda_{c}$ baryons have a large probability of becoming $D$ mesons after a couple of interactions. This may actually be an important source of $D$ mesons in extensive showers if the intrinsic charm [1] dominates the PDFs at high energy in proton collisions.

The proper inclusion of charm in extensive air-shower simulators should describe both its production (already in DPMJET [2]) and also its propagation. $D$ and $\Lambda_{c}$ hadronic collisions are obviously irrelevant in colliders, which explains why they are absent in codes like SIBYLL [11] or QGSJET [13]. We think, however, that the possibility to search for observable effects in astroparticle experiments should make worthy an effort in such direction.

\section{Acknowledgments}

We would like to thank Paolo Lipari, Davide Meloni and Sergio Sciutto for discussions. This work has been partially supported by MICINN of Spain (FPA2010-16802, FPA2006-05294, and Consolider-Ingenio Multidark CSD2009-00064) and by Junta de Andalucía (FQM 101 and FQM 437).

\section{References}

[1] S. J. Brodsky, P. Hoyer, C. Peterson and N. Sakai, Phys. Lett. B 93 (1980) 451. 
[2] P. Berghaus, T. Montaruli and J. Ranft, JCAP 0806 (2008) 003 arXiv:0712.3089 [hep$\mathrm{ex}]$.

[3] P. Gondolo, G. Ingelman and M. Thunman, Astropart. Phys. 5 (1996) 309 arXiv:hep-ph/9505417].

[4] C. G. S. Costa, Astropart. Phys. 16 (2001) 193 [arXiv:hep-ph/0010306].

[5] J. I. Illana, M. Masip and D. Meloni, JCAP 0909 (2009) 008 [arXiv:0907.1412 [hep-ph]].

[6] J. I. Illana, P. Lipari, M. Masip and D. Meloni, arXiv:1010.5084 [astro-ph.HE].

[7] H. Baer, K. m. Cheung and J. F. Gunion, Phys. Rev. D 59 (1999) 075002 arXiv:hep-ph/9806361].

[8] J. I. Illana, M. Masip and D. Meloni, Phys. Rev. D 75 (2007) 055002 arXiv:hep-ph/0611036.

[9] T. Sjostrand, S. Mrenna and P. Z. Skands, JHEP 0605 (2006) 026 arXiv:hep-ph/0603175.

[10] S. Navin, "Diffraction in Pythia," arXiv:1005.3894 [hep-ph].

[11] E. J. Ahn, R. Engel, T. K. Gaisser, P. Lipari and T. Stanev, Phys. Rev. D 80 (2009) 094003 arXiv:0906.4113 [hep-ph]].

[12] S. Ostapchenko, Nucl. Phys. Proc. Suppl. 196 (2009) 90.

[13] S. Ostapchenko, AIP Conf. Proc. 928 (2007) 118 [arXiv:0706.3784 [hep-ph]]. 